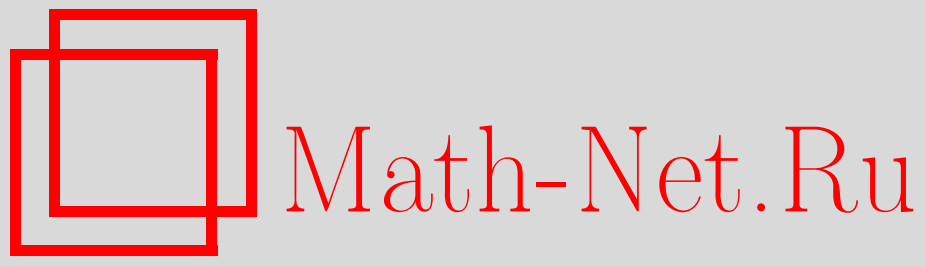

Е. Л. Лакштанов, Р. А. Минлос, Спектр двухчастичных связанных состояний трансфер-матриц гиббсовских полей (уединенное связанное состояние), Функи. анализ и его прил., 2004, том 38, выпуск $3,52-69$

DOI: https://doi.org/10.4213/faa117

Использование Общероссийского математического портала MathNet.Ru подразумевает, что вы прочитали и согласны с пользовательским соглашением

http://www.mathnet.ru/rus/agreement

Параметры загрузки:

IP: 3.89 .185 .249

26 апреля 2023 г., 15:18:29

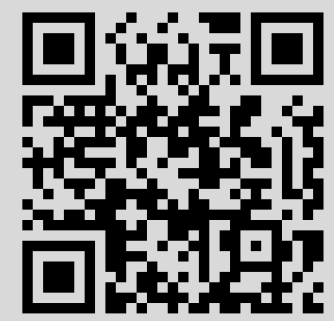


Функииональный анализ и его приложения

2004, т. 38, вып. 3, с. 52-69

УДК 517.9

\title{
Спектр двухчастичных связанных состояний трансфер-матриц гиббсовских полей (уединенное связанное состояние)
}

\author{
(C) 2004. Е. Л. ЛАКштанов, Р. А. МинЛос
}

Посвящается В. Б. Лидскому в связи с его восъмидесятилетием

\section{§1. Введение и постановка задачи}

Изучение трансфер-матриц (стохастических операторов) многих решетчатых моделей статистической физики и квантовой теории поля (в высокотемпературной области) приводит к так называемой «корпускулярной картине» их спектра. Это означает, что в гильбертовом пространстве функций от поля, в котором действует трансфер-матрица $\mathscr{T}_{\beta}(\beta=1 / T)$, можно выделить некоторые специальные подпространства, инвариантные относительно трансфер-матрицы и группы трансляций (в предположении трансляционной инвариантности модели): одночастичное, двухчастичное, трехчастичное и т. д. подпространства (см. об этом подробнее в $[1,2])$. После этого возникает задача об изучении спектра трансферматрицы в каждом из этих подпространств. Если спектр в одночастичном подпространстве описывается сравнительно легко, то уже в двухчастичном подпространстве изучение этого спектра является непростой задачей из-за наличия так называемых «связанных состояний». Чисто аналитически эта задача состоит в нахождении собственных значений некоторого семейства операторов фридрихсовского типа. В таком виде, применительно к случаю трансфер-матрицы модели Изинга, задача была сформулирована (и решена для двумерной модели Изинга) в работах [3-5]. Более общий случай, охватывающий, в частности, модель Изинга произвольной размерности, изучался в работах $[1,6]$. Здесь обобщаются результаты упомянутых работ. Это обобщение позволяет изучать так называемые двухчастичные связанные состояния трансфер-матриц для гиббсовских полей с компактным пространством спинов более общего вида, чем в модели Изинга, и с взаимодействием между спинами также общего вида (произвольное финитное взаимодействие вдоль «пространственных» направлений).

В упомянутой работе [5], где впервые было предпринято такое обобщение, как и в данной работе, рассматриваются лишь потенциалы «общего положения». Для таких потенциалов свойства соответствующего фридрихсовского семейства операторов сохраняются при малых «шевелениях» потенциала или что одно и тоже - при малых шевелениях «символа» этого семейства (т. е. энергии двух «квазичастиц» в состоянии рассеяния как функции их квазиимпульсов). Потенциалы взаимодействия, приводящие к исключительным «символам», образуют гиперповерхность конечной коразмерности в пространстве всех потенциалов. Следует отметить, что часто рассматриваемый случай взаимодействия 
ближайших соседей приводит к символам уже не общего положения, и тогда картина спектра отличается от той, которая описана в данной статье.

В настоящей работе мы следуем плану статьи [5]; при этом мы рассматриваем более общие спиновые пространства и приводим подробные доказательства, которые в [5] лишь кратко намечены. Работа состоит из нескольких частей. В данной, первой ее части мы описываем изучаемые модели в общем виде для любого значения размерности решетки и устанавливаем существование так называемого «уединенного уровня» (уточняя при этом результаты работ $[9,11]$ ), находящегося на расстоянии порядка $\beta^{2}$ от непрерывного спектра.

Кроме этого уровня, существующего при всех значениях полного квазиимпульса системы, могут существовать другие связанные состояния, так называемые «прилегающие» уровни, «живущие» лишь в окрестностях некоторых специальных значений полного квазиимпульса и расположенные на расстояниях порядка $\beta^{\alpha}, \alpha \geqslant 4$, от краев непрерывного спектра. Эти прилегающие уровни мы изучим в следующих частях предлагаемой работы.

Итак, мы предполагаем, что формальный гамильтониан гиббсовского поля имеет вид

$$
H(\sigma)=\beta \sum \Phi(x-y) \sigma(x) \sigma(y)
$$

где $\sigma=\left\{\sigma(x), x \in \mathbb{Z}^{\nu} \times \mathbb{Z}\right\}\left(\mathbb{Z}^{\nu}\right.$ - «пространство», $\mathbb{Z}$ - «время») - конфигурация поля, принимающая значения в некотором компактном симметричном относительно нуля подмножестве $S \in \mathbb{R}^{1}$, причем $\# S>2$.

Здесь $\beta=1 / T$ - обратная температура (которая в дальнейшем предполагается достаточно малой), а $\Phi(z)$ - финитный потенциал взаимодействия, отличный от нуля только для векторов вида $z=\left(\bar{z}, z_{0}\right) \in \mathbb{Z}^{\nu} \times \mathbb{Z}^{1}$, у которых «временна́я» координата удовлетворяет условию $\left|z_{0}\right| \leqslant 1$ (это условие и позволяет рассматривать наше гиббсовское поле как марковское вдоль направления «времени»). При этом в данной работе мы рассматриваем даже более узкий класс потенциалов $\Phi(z)$ : их носители состоят либо из векторов $(\bar{z}, 0)$, либо из векторов $\left(0, z_{0}\right),\left|z_{0}\right|=1$.

Как обычно, предполагается, что задано некоторое симметричное относительно нуля четное распределение вероятностей $v$ на множестве $S$, с помощью которого задается «свободная» мера $\mu_{0}=v^{\mathbb{Z}^{\nu+1}}$ в пространстве $\Omega=S^{\mathbb{Z}^{\nu+1}}$ всех конфигураций поля. Гиббсовское поле $\mu_{\beta}$ определяется стандартным образом с помощью гамильтониана (1.1) и свободной меры $\mu_{0}$ (подробности см., например, в [7]). Относительно меры $v$ мы сделаем следующее предположение:

$$
\left\langle\sigma^{4}\right\rangle_{v} \neq 3\left\langle\sigma^{2}\right\rangle_{v}
$$

где $\left\langle\sigma^{k}\right\rangle_{v}=\int_{S} \sigma^{k} d v(\sigma)$ есть $k$-й момент распределения $v$.

Далее, рассматривается так называемое физическое пространство $\mathscr{H} \subset$ $L_{2}\left(\Omega, \mu_{\beta}\right)$, состоящее из функций $f(\sigma)$, зависящих лишь от значений конфигурации $\sigma$ в нулевой момент времени. В $\mathscr{H}$ обычным образом вводится стохастический оператор гиббсовского поля $\mathscr{T}_{\beta}$ (называемый обычно трансфер-матрицей). Поскольку мера $\mu_{\beta}$ инвариантна относительно пространственных сдвигов поля, в пространстве $\mathscr{H}$ определена также унитарная группа $\left\{U_{t}, t \in \mathbb{Z}^{\nu}\right\}$ пространственных трансляций, коммутирующая с $\mathscr{T}_{\beta}$ (подробнее см. [2]).

Напомним основную схему построения одночастичного и двучастичного инвариантных пространств трансфер-матрицы $\mathscr{T}_{\beta}$ (и группы трансляций $U_{t}$ ). В качестве исходного приближения к одночастичному пространству выбирается 
линейная оболочка функций $\left\{h_{x}^{0}(\sigma)=\sigma(x), x \in \mathbb{Z}^{\nu}\right\}$, и затем с помощью некоторого приема теории возмущений (см. [1,2]) строится истинное инвариантное подпространство $\mathscr{H}_{1}$ вместе с ортонормированным базисом $\left\{h_{x}, x \in \mathbb{Z}^{\nu}\right\}$, в котором операторы трансляций $\left.U_{t}\right|_{\mathscr{H}_{1}}=U_{t}^{(1)}$ действуют циклически, $U_{t}^{(1)} h_{x}=h_{x+t}$, $t, x \in \mathbb{Z}^{\nu}$, а трансфер-матрица $\left.\mathscr{T}_{\beta}\right|_{\mathscr{H}_{1}}=\mathscr{T}_{\beta}^{(1)}-$ с помощью свертки:

$$
\mathscr{T}_{\beta}^{(1)} h_{x}=\sum_{y \in C_{1}} a_{x-y} h_{y}
$$

с некоторой экспоненциально убывающей функцией $\left\{a_{z}, z \in \mathbb{Z}^{\nu}\right\}$.

После унитарного отображения («преобразования Фурье»)

$$
W_{1}: \mathscr{H}_{1} \rightarrow L_{2}\left(\mathbb{T}^{\nu}, d \lambda\right), \quad\left(W_{1} h_{x}\right)(\lambda)=e^{i(\lambda, x)}, \quad \lambda \in \mathbb{T}^{\nu},
$$

где $\mathbb{T}^{\nu}=[0,2 \pi]^{\nu}$ есть $\nu$-мерный тор, а $d \lambda$ - нормированная мера Хаара на $\mathbb{T}^{\nu}$, операторы $U_{t}^{(1)}$ и $\mathscr{T}_{\beta}^{(1)}$ перейдут в операторы $\widetilde{U}_{t}^{(1)}, \widetilde{\mathscr{T}}_{\beta}^{(1)}$ умножения на функции:

$$
\left(\widetilde{U}_{t}^{(1)} f\right)(\lambda)=e^{i(t, \lambda)} f(\lambda), \quad\left(\widetilde{\mathscr{T}}_{\beta}^{(1)} f\right)(\lambda)=\tilde{a}(\lambda) f(\lambda), \quad f \in L_{2}\left(\mathbb{T}^{\nu}, d \lambda\right), \lambda \in \mathbb{T}^{\nu}, t \in \mathbb{Z}^{\nu},
$$

где $\tilde{a}(\lambda)=\sum_{z} a_{z} e^{i(z, \lambda)}-$ преобразование Фурье функции $a$.

ЗАмЕчАниЕ. Согласно физической интерпретации, оператор $-\frac{1}{2} \ln \mathscr{T}_{\beta}^{2}$ объявляется гамильтонианом соответствующего решетчатого поля, а пространство $\mathscr{H}_{1}$ - пространством состояний его «квазичастицы» (с наименьшей массой), причем $\lambda$ - квазиимпульс этой «квазичастицы», а $-\frac{1}{2} \ln \tilde{a}^{2}(\lambda)-$ энергия «квазичастицы» с квазиимпульсом $\lambda$.

В силу того, что функция $a_{x}$ убывает экспоненциально, с большим показателем экспоненты, функция $\tilde{a}(\lambda)$ аналитична в некоторой комплексной окрестности тора $\mathbb{T}^{\nu}$

$$
W_{A}=\left\{\lambda \in \mathbb{C}^{\nu} / \mathbb{Z}^{\nu}:\left|\operatorname{Im} \lambda^{(i)}\right|<A, i=1, \ldots, \nu\right\},
$$

где значение $A=A(\beta)$ достаточно велико при малых $\beta$ (через $\mathbb{C}^{\nu} / \mathbb{Z}^{\nu}$ обозначено факторпространство относительно действия $z \rightarrow z+n, z \in \mathbb{C}^{\nu}, n \in \mathbb{Z}^{\nu}$, группы $\mathbb{Z}^{\nu}$ в $\left.\mathbb{C}^{\nu}\right)$. Кроме того, $\tilde{a}(\lambda)$ аналитически зависит от $\beta$ и при малых $\beta$ допускает представление

$$
\tilde{a}(\lambda)=\beta a_{0}+\beta^{2} a_{1}(\lambda)+a_{2}(\lambda, \beta),
$$

где $a_{2}(\lambda, \beta) \sim O\left(\beta^{3}\right)$, а константа $a_{0} \neq 0$ и функция $a_{1}(\lambda)$ обе не зависят от $\beta$. Здесь и в дальнейшем обозначение $O\left(\beta^{k}\right)$ означает, что соответствующая величина допускает оценку $C \beta^{k}$ с константой $C>0$, не зависящей от $\beta$ (и, возможно, других переменных).

Двухчастичное пространство оператора $\mathscr{T}_{\beta}$ строится подобным же образом: в качестве приближения к нему выбирается пространство, натянутое на векторы $\left\{\sigma(x) \sigma(y),(x, y) \in \mathbb{Z}^{\nu} \times \mathbb{Z}^{\nu}, x \neq y ; \sigma^{2}(x), x \in \mathbb{Z}^{\nu}\right\}$, и затем, применяя упомянутый выше прием из теории возмущений, мы получаем инвариантное относительно $\mathscr{T}_{\beta}$ и $\left\{U_{t}\right\}$ подпространство $\mathscr{H}_{2}$ вместе с некоторым его специальным базисом

$$
\left\{h_{x}^{(2)}, x \in \mathbb{Z}^{\nu} ; h_{(x, y)},(x, y) \in \mathbb{Z}^{\nu} \times \mathbb{Z}^{\nu}, x \neq y\right\} .
$$

ЗАмечание. В случае модели Изинга $\sigma_{x}^{2} \equiv 1$ и поэтому в $\mathscr{H}_{2}$ отсутствует часть $\left\{h_{x}^{(2)}, x \in \mathbb{Z}^{\nu}\right\}$ базиса. 
Действие операторов $U_{t}^{(2)}=\left.U_{t}\right|_{\mathscr{H}_{2}}$ и $\mathscr{T}_{\beta}^{(2)}=\left.\mathscr{T}_{\beta}\right|_{\mathscr{H}_{2}}$ удобнее сразу описать после «преобразования Фурье»

$$
W_{2}: \mathscr{H}_{2} \rightarrow L_{2}\left(\mathbb{T}^{\nu}, d \lambda\right) \oplus \bar{L}_{2}^{\mathrm{sym}}\left(\mathbb{T}^{\nu} \times \mathbb{T}^{\nu}, d \lambda_{1} d \lambda_{2}\right) \equiv \widetilde{\mathscr{H}}_{2}
$$

где $\bar{L}_{2}^{\text {sym }}\left(\mathbb{T}^{\nu} \times \mathbb{T}^{\nu}, d \lambda_{1} d \lambda_{2}\right)$ - гильбертово пространство симметрических функций $f\left(\lambda_{1}, \lambda_{2}\right), \lambda_{1}, \lambda_{2} \in \mathbb{T}^{\nu}$, ортогональных любой функции вида $h\left(\lambda_{1}+\lambda_{2}\right)$, $h(\lambda) \in L_{2}\left(\mathbb{T}^{\nu}, d \lambda\right)$, а отображение $W_{2}$ задается формулами

$$
\begin{aligned}
\left(W_{2} h_{x}^{(2)}\right)(\lambda) & =e^{i(x, \lambda)} \in L_{2}\left(\mathbb{T}^{\nu}, d \lambda\right), \\
\left(W_{2} h_{(x, y)}\right)\left(\lambda_{1}, \lambda_{2}\right) & =\frac{1}{\sqrt{2}}\left(e^{i\left(\lambda_{1}, x\right)+i\left(\lambda_{2}, y\right)}+e^{i\left(\lambda_{1}, y\right)+i\left(\lambda_{2}, x\right)}\right) \in \bar{L}_{2}^{\mathrm{sym}}\left(\mathbb{T}^{\nu} \times \mathbb{T}^{\nu}, d \lambda_{1} d \lambda_{2}\right) .
\end{aligned}
$$

При этом операторы $\widetilde{U}_{t}^{(2)}$ и $\widetilde{\mathscr{T}}_{\beta}^{(2)}$ в пространстве $\widetilde{\mathscr{H}}_{2}$ (получаемые из $U_{t}^{(2)}$ и $\mathscr{T}_{\beta}^{(2)}$ при отображении $\left.W_{2}\right)$ имеют вид

$$
\begin{aligned}
& \left(\widetilde{U}_{t}^{(2)} F\right)_{1}(\lambda)=e^{i(\lambda, t)} f_{1}(\lambda), \quad\left(\widetilde{U}_{t}^{(2)} F\right)_{2}\left(\lambda_{1}, \lambda_{2}\right)=e^{i\left(\lambda_{1}+\lambda_{2}, t\right)} f_{2}\left(\lambda_{1}, \lambda_{2}\right), \\
& \left(\widetilde{\mathscr{T}}_{t}^{(2)} F\right)_{1}(\lambda)=\bar{\gamma}(\lambda) f_{1}(\lambda)+\int_{\mathbb{T}^{\nu} \times \mathbb{T}^{\nu}} b\left(\lambda, \mu_{1}, \mu_{2}\right) \delta\left(\lambda-\mu_{1}-\mu_{2}\right) f_{2}\left(\mu_{1}, \mu_{2}\right) d \mu_{1} d \mu_{2} \\
& \left(\widetilde{\mathscr{T}}_{t}^{(2)} F\right)_{2}\left(\lambda_{1}, \lambda_{2}\right)=\tilde{a}\left(\lambda_{1}\right) \tilde{a}\left(\lambda_{2}\right) f_{2}\left(\lambda_{1}, \lambda_{2}\right) \\
& \quad+\int_{\left(\mathbb{T}^{\nu}\right)^{2}}\left(S\left(\lambda_{1}, \lambda_{2}, \mu_{1}, \mu_{2}\right)-\tilde{a}\left(\mu_{1}\right) \tilde{a}\left(\mu_{2}\right)\right) \delta\left(\lambda_{1}+\lambda_{2}-\mu_{1}-\mu_{2}\right) f_{2}\left(\mu_{1}, \mu_{2}\right) d \mu_{1} d \mu_{2} \\
& \quad+\int_{\mathbb{T}^{\nu}} b^{*}\left(\lambda_{1}, \lambda_{2}, \mu\right) \delta\left(\lambda_{1}+\lambda_{2}-\mu\right) f_{1}(\mu) d \mu
\end{aligned}
$$

где $F=\left(f_{1}(\lambda), f_{2}\left(\lambda_{1}, \lambda_{2}\right)\right) \in L_{1}\left(\mathbb{T}^{\nu}\right) \oplus \bar{L}_{2}^{\text {sym }}\left(\mathbb{T}^{\nu} \times \mathbb{T}^{\nu}\right)$, а $\delta(\cdot)$ - дельта-функция на торе $\mathbb{T}^{\nu}$.

Здесь $\gamma(\lambda)$ - четная вещественная функция, аналитическая в комплексной окрестности (1.4) тора $\mathbb{T}^{\nu}, \tilde{a}(\lambda)$ - та же функция, что и в (1.3), а $S\left(\lambda_{1}, \lambda_{2}, \mu_{1}, \mu_{2}\right)$ определена на гиперповерхности $\Gamma=\left\{\lambda_{1}+\lambda_{2}=\mu_{1}+\mu_{2}\right\} \subset\left(\mathbb{T}^{\nu}\right)^{4}$ и также аналитична в комплексной окрестности этой гиперповерхности. При этом выполняется условие самосопряженности

$$
S\left(\lambda_{1}, \lambda_{2}, \mu_{2}, \mu_{2}\right)=\overline{S\left(\mu_{1}, \mu_{2}, \lambda_{1}, \lambda_{2}\right)},
$$

а также условие

$$
\int S\left(\lambda_{1}, \lambda_{2}, \mu_{1}, \mu_{2}\right) \delta\left(\lambda_{1}+\lambda_{2}-\mu_{1}-\mu_{2}\right) d \mu_{1} d \mu_{2}=0 .
$$

Кроме того, $S\left(\lambda_{1}, \lambda_{2}, \mu_{1}, \mu_{2}\right)$ является симметрической функцией как по первой паре переменных $\left(\lambda_{1}, \lambda_{2}\right)$, так и по второй паре $\left(\mu_{1}, \mu_{2}\right)$.

Наконец, ядра $b\left(\lambda, \mu_{1}, \mu_{2}\right)$ и $b^{*}\left(\lambda_{1}, \lambda_{2}, \mu\right)$, определенные соответственно на гиперповерхностях $\left\{\lambda=\mu_{1}+\mu_{2}\right\}$ и $\left\{\lambda_{1}+\lambda_{2}=\mu\right\}$, также аналитичны и связаны соотношениями

$$
b^{*}\left(\lambda_{1}, \lambda_{2}, \mu\right)=\overline{b\left(\mu, \lambda_{1}, \lambda_{2}\right)}, \quad \int b\left(\lambda, \mu_{1}, \mu_{2}\right) \delta\left(\lambda-\mu_{1}-\mu_{2}\right) d \mu_{1} d \mu_{2}=0 .
$$

Функция $b^{*}\left(\lambda_{1}, \lambda_{2}, \mu\right)$ является симметрической по паре переменных $\left(\lambda_{1}, \lambda_{2}\right)$, a функция $b\left(\lambda, \mu_{1}, \mu_{2}\right)$ - по паре $\left(\mu_{1}, \mu_{2}\right)$. 
Все перечисленные свойства входящих в (1.8), (1.9) функций обеспечивают самосопряженность оператора $\widetilde{\mathscr{T}}_{\beta}^{(2)}$ в пространстве $\widetilde{\mathscr{H}}_{2}$. Кроме того, все эти функции аналитически зависят от $\beta$ и при малых $\beta$ допускают разложения

$$
\begin{aligned}
\bar{\gamma}(\lambda) & =\beta^{2}\left(\gamma_{0}+O\left(\beta^{2}\right)\right), \\
\tilde{a}\left(\lambda_{1}\right) \tilde{a}\left(\lambda_{2}\right) & =\beta^{2}\left(a_{0}^{2}+2 \beta a_{0} a_{1}(\lambda)+O\left(\beta^{2}\right)\right), \\
S\left(\lambda_{1}, \lambda_{2}, \mu_{1}, \mu_{2}\right) & =\beta^{2}\left(\beta^{2} S_{0}\left(\lambda_{1}, \lambda_{2}, \mu_{1}, \mu_{2}\right)+O\left(\beta^{3}\right)\right), \\
b\left(\lambda_{1}, \lambda_{2}, \mu\right) & =\beta^{2}\left(\beta b_{0}\left(\lambda_{1}, \lambda_{2}, \mu\right)+O\left(\beta^{2}\right)\right),
\end{aligned}
$$

где $\gamma_{0}, S_{0}$ и $b_{0}$ не зависят от $\beta$. Естественно сократить все эти выражения на $\beta^{2}$ и впредь рассматривать оператор $\widehat{\mathscr{T}}_{\beta}^{(2)}=\beta^{-2} \widetilde{\mathscr{T}}_{\beta}^{(2)}$.

Вся изложенная здесь вкратце схема построения одно- и двухчастичных инвариантных подпространств трансфер-матрицы, а также вид трансфер-матрицы в этих подпространствах описаны в книге [2] и статьях $[9,10]$.

В этой работе мы будем исследовать спектр оператора $\mathscr{T}$ в пространстве $\widetilde{\mathscr{H}}_{2}$, введенном в (1.5), чуть более общего вида, чем оператор $\widehat{\mathscr{T}}_{\beta}^{(2)}$, а именно в выражении (1.9) умножение на $\tilde{a}\left(\lambda_{1}\right) \tilde{a}\left(\lambda_{2}\right)$ мы заменим умножением на функцию более общего вида $\beta^{2} \omega\left(\lambda_{1}, \lambda_{2}\right)$, причем для $\omega$ выполняется условие (аналогичное (1.13) после сокращения на $\beta^{2}$ )

$$
\omega\left(\lambda_{1}, \lambda_{2}\right)=\omega_{0}+\beta \omega_{1}\left(\lambda_{1}, \lambda_{2}\right)+\omega_{2}\left(\lambda_{1}, \lambda_{2}\right),
$$

где константа $\omega_{0}$ и функция $\omega_{1}\left(\lambda_{1}, \lambda_{2}\right)$ обе не зависят от $\beta$, а $\omega_{2}\left(\lambda_{1}, \lambda_{2}\right)=O\left(\beta^{2}\right)$. При этом предполагается, что $\omega\left(\lambda_{1}, \lambda_{2}\right)$ аналитична по каждой переменной $\lambda_{1}$ и $\lambda_{2}$ в области (1.4).

Поскольку, как видно из $(1.8),(1.9)$, оператор $\widehat{\mathscr{T}}_{\beta}^{(2)}$ сохраняет полный квазиимпульс $\Lambda=\lambda_{1}+\lambda_{2}$, естественно будет перейти в (1.8) и (1.9) к переменным $\Lambda=\lambda_{1}+\lambda_{2}$ и $k=\lambda_{1}$ и представить пространство $\widetilde{\mathscr{H}_{2}}$ в виде прямого интеграла (cм. $[12])$,

$$
\widetilde{\mathscr{H}_{2}}=\int \mathscr{H}_{\Lambda}^{(2)} d \Lambda
$$

где $\mathscr{H}_{\Lambda}^{(2)}=\mathbb{C}^{1} \oplus \bar{L}_{2}^{\Lambda}\left(\mathbb{T}^{\nu}\right)$, а $\bar{L}_{2}^{\Lambda}\left(\mathbb{T}^{\nu}\right) \subset L_{2}\left(\mathbb{T}^{\nu}\right)-$ пространство функций $\{f(k)$, $\left.k \in \mathbb{T}^{\nu}\right\}$, инвариантных относительно инволюции тора $\mathbb{T}^{\nu}: k \rightarrow \Lambda-k$ и таких, что $\int f(k) d k=0$. При этом оператор $\mathscr{T}$ также разложится в прямой интеграл операторов,

$$
\mathscr{T}=\int_{\mathbb{T}^{\nu}} \mathscr{T}_{\Lambda} d \Lambda
$$

где $\mathscr{T}_{\Lambda}$ действует на векторы $F=\left(c_{0}, f\right) \in \mathscr{H}_{\Lambda}^{(2)}$ по формулам

$$
\begin{aligned}
\left(\mathscr{T}_{\Lambda} F\right)_{0} & =\gamma(\Lambda) c_{0}+\int b_{\Lambda}(p) f(p) d p \\
\left(\mathscr{T}_{\Lambda} F\right)_{1}(k) & =\omega_{\Lambda}(k) f(k)+\int\left(K_{\Lambda}(k, p)-\omega_{\Lambda}(p)\right) f(p) d p+b_{\Lambda}^{*}(k) c_{0} .
\end{aligned}
$$


Здесь

$$
\begin{aligned}
\gamma & =\beta^{-2} \bar{\gamma}=\gamma_{0}+O\left(\beta^{2}\right), \\
\omega_{\Lambda}(k) & =\omega(k, \Lambda-k), \\
K_{\Lambda}(k, p) & =\beta^{-2} S(k, \Lambda-k, p, \Lambda-p)=\beta^{2} S_{0}(k, \Lambda-k, p, \Lambda-p)+O\left(\beta^{3}\right), \\
b_{\Lambda}(p) & =\beta^{-2} b_{0}(\Lambda, p, \Lambda-p)=\beta b_{0}(\Lambda, p, \Lambda-p)+O\left(\beta^{2}\right), \\
b_{\Lambda}^{*}(k) & =\beta^{-2} b_{0}^{*}(k, \Lambda-k, \Lambda)=\beta b_{0}^{*}(k, \Lambda-k, \Lambda)+O\left(\beta^{2}\right) .
\end{aligned}
$$

Заметим, что условия (1.10)-(1.12) влекут за собой следующие соотношения:

$$
\int K_{\Lambda}(k, p) d p=0, \quad \int b_{\Lambda}(p) d p=0, \quad K_{\Lambda}(k, p)=\bar{K}_{\Lambda}(p, k), \quad b_{\Lambda}^{*}(k)=\bar{b}_{\Lambda}(k),
$$

а также, что очень важно, соотношение

$$
\omega_{\Lambda}(k)=\omega_{\Lambda}(\Lambda-k)
$$

для $\omega_{\Lambda}(k)$ и соотношения $K_{\Lambda}(k, p)=K_{\Lambda}(\Lambda-k, p), K_{\Lambda}(k, p)=K_{\Lambda}(k, \Lambda-p)$. Кроме того, $b_{\Lambda}(k)$ и $b_{\Lambda}^{*}(p)$ не меняются при заменах $k \rightarrow \Lambda-k, p \rightarrow \Lambda-p$. Для нас существенно, что при любом $\Lambda \in \mathbb{T}^{\nu}$ функции $\omega_{\Lambda}(k)$ и $b_{\Lambda}(k)$ аналитичны относительно $k$ в комплексной окрестности (1.4) тора $\mathbb{T}^{\nu}$, а ядро $K_{\Lambda}(k, p)$ также аналитично по $k$ и $p$ в декартовом произведении этих окрестностей.

Итак, мы пришли к задаче изучения спектра семейства операторов $\left\{\mathscr{T}_{\Lambda}\right.$, $\left.\Lambda \in \mathbb{T}^{\nu}\right\}$ вида (1.15). Операторы $\mathscr{T}_{\Lambda}$ представляют собой некоторое обобщение операторов Фридрихса (см. [8,13]), и известно, что при наших условиях относительно входящих в (1.15) функций каждый оператор $\mathscr{T}_{\Lambda}$ имеет отрезок непрерывного (лебеговского) спектра $\left[\min _{k} \omega_{\Lambda}(k), \max _{k} \omega_{\Lambda}(k)\right]$ и еще, быть может, конечное число изолированных собственных значений, лежащих как вне непрерывного спектра, так и, быть может, на непрерывном спектре. Последний случай возникает редко и требует отдельного рассмотрения. Таким образом, задача этой статьи и последующих - изучить при малых значениях параметра $\beta$ поведение собственных значений семейства операторов $\mathscr{T}_{\Lambda}$, лежащих вне непрерывного спектра этих операторов.

Собственные значения $\varepsilon_{i}(\Lambda)$ оператора $\mathscr{T}_{\Lambda}$, лежащие вне непрерывного спектра, непрерывно меняясь при изменении $\Lambda$, «живут» в целой области $G_{i} \subset \mathbb{T}^{\nu}$ значений $\Lambda$ (т. е. $\varepsilon_{i}(\Lambda)$ существует, только когда $\Lambda$ принадлежит некоторой области $\left.G_{i}\right)$. В случае, когда $G_{i} \neq \mathbb{T}^{\nu}$, на границе $\partial G_{i}$ области $G_{i}$ собственное значение $\varepsilon_{i}(\Lambda)$ «сливается» с краем непрерывного спектра и поглощается им.

Можно показать (см. например $[9,11])$, что условие $(1.2)$ влечет за собой условие

$$
\gamma_{0} \neq \omega_{0}
$$

из которого, в частности, следует, что при малых $\beta$ при каждом $\Lambda$ значение $\gamma(\Lambda)$ лежит вне непрерывного спектра оператора $\mathscr{T}_{\Lambda}$.

\section{§2. Уединенное собственное значение оператора $\mathscr{T}_{\Lambda}$}

В этом параграфе мы начинаем исследование собственных значений операторов $\mathscr{T}_{\Lambda}$. Мы сформулируем здесь общий результат относительно так называемого «уединенного» собственного значения оператора $\mathscr{T}_{\Lambda}$, верный для любой размерности $\nu$. 
ТЕОРема 2.1. При выполнении всех указанных выше условий у оператора $\mathscr{T}_{\Lambda}$ при всех $\Lambda$ существует единственное собственное значение $\varepsilon_{b}(\Lambda)$, имеющее вид

$$
\varepsilon_{b}(\Lambda)=\gamma_{0}+O\left(\beta^{2}\right)
$$

(т.е. $\left|\varepsilon_{b}(\Lambda)-\gamma_{0}\right|<C \beta^{2}$ при некоторой константе $C>0$, не зависящей от $\beta$ $u \Lambda)$.

ЗАМЕЧАниЕ. В силу условий $(1.13),(1.14),(1.19)$ собственное значение $\varepsilon_{b}(\Lambda)$ находится на конечном расстоянии от непрерывного спектра оператора $\mathscr{T}_{\Lambda}$, т. е.

$$
\min _{\Lambda, \text { ext }=\text { min,max }}\left|\varepsilon_{b}(\Lambda)-\tau_{\Lambda}^{\text {ext }}\right|>\tau>0,
$$

где $\tau>0$ не зависит от $\beta, \Lambda$, a $\tau_{\Lambda}^{\text {ext }}$, ext $=\max , \min$, означает максимальное или минимальное значение функции $\omega_{\Lambda}$.

ЗАмечАниЕ. Обозначим через $R_{\Lambda} \subset \mathscr{H}_{\Lambda}^{(2)}$ одномерное собственное подпространство оператора $\mathscr{T}_{\Lambda}$ с собственным значением $\varepsilon_{b}(\Lambda)$, и пусть

$$
\widehat{\mathscr{H}}_{1}=\int_{\mathbb{T}^{\nu}} R_{\Lambda} d \Lambda
$$

- подпространство пространства $\widetilde{\mathscr{H}}_{2}$, которое можно отождествить с пространством $L_{2}\left(\mathbb{T}^{\nu}, d \Lambda\right)$. Очевидно, что это подпространство инвариантно относительно $\mathscr{T}$ и группы $U_{t}$, которые действуют по формулам

$$
(\mathscr{T} f)(\Lambda)=\varepsilon_{b}(\Lambda) f(\Lambda), \quad\left(U_{t} f\right)(\Lambda)=e^{i(t, \Lambda)} f(\Lambda), \quad f \in \widehat{\mathscr{H}}_{1} .
$$

Таким образом, $\widehat{\mathscr{H}}_{1}$ имеет структуру одночастичного подпространства, т. е. является пространством состояний некоторой новой «квазичастицы» поля с энергией $-\ln \left|\varepsilon_{b}(\Lambda)\right|$.

Отметим, что существование пространства $\widehat{\mathscr{H}}_{1}$ для спиновых систем с пространством спинов $S, \# S>2$, было установлено в работах $[9,11]$ (частный случай рассмотрен в [10]).

Остальные собственные значения оператора $\mathscr{T}_{\Lambda}$, лежащие вне его непрерывного спектра, как мы увидим, могут находиться лишь поблизости от одного из его краев $\tau_{\Lambda}^{\min }$ или $\tau_{\Lambda}^{\max }$, т. е. на расстоянии порядка $\beta^{\alpha}, \alpha>0$, от одного из них. Все эти собственные значения оператора $\mathscr{T}_{\Lambda}$ условимся называть «прилегающими уровнями».

ДокАЗАТЕЛЬСТво тЕОРемы 2.1. Собственный вектор $F=(c, f), c \in \mathbb{C}^{1}$, $f \in \bar{L}_{2}^{\Lambda}\left(\mathbb{T}^{\nu}\right)$, оператора $\mathscr{T}_{\Lambda}$ с собственным значением $z$, согласно $(1.15)$, удовлетворяет уравнениям

$$
\left\{\begin{array}{l}
(\gamma(\Lambda)-z) c+\int_{\mathbb{T}^{\nu}} f(\mu) b_{\Lambda}(\mu) d \mu=0, \\
\left(\omega_{\Lambda}(k)-z\right) f(k)-\int_{\mathbb{T}^{\nu}} \omega_{\Lambda}(\mu) f(\mu) d \mu+\int_{\mathbb{T}^{\nu}} K_{\Lambda}(k, \mu) f(\mu) d \mu+\bar{b}_{\Lambda}(k) c=0 .
\end{array}\right.
$$

Отсюда, выражая $c$ из первого уравнения и подставляя полученное выражение во второе, получим, что

$$
\left(\omega_{\Lambda}(k)-z\right) f(k)-\int_{\mathbb{T}^{\nu}} \omega_{\Lambda}(\mu) f(\mu) d \mu+\int_{\mathbb{T}^{\nu}} M_{\Lambda}(k, \mu ; z) f(\mu) d \mu=0,
$$


где $M_{\Lambda}(k, \mu ; z)=K_{\Lambda}\left(k_{1}, k_{2}\right)-b_{\Lambda}\left(k_{1}\right) \bar{b}_{\Lambda}\left(k_{2}\right) /(\gamma(\Lambda)-z)$.

ЛЕмма 2.1. Рассмотрим интегральное уравнение относительно функиии $f \in \bar{L}_{2}\left(\mathbb{T}^{\nu}\right)=(\text { const })^{\perp} \subset L_{2}\left(\mathbb{T}^{\nu}\right)$

$$
(\omega(k)-z) f(k)-\int_{\mathbb{T}^{\nu}} \omega(p) f(p) d p+\int_{\mathbb{T}^{\nu}} L(k, p ; z) f(p) d p=0,
$$

где $L(k, p ; z)$ - функиия, гладкая по $(k, p) \in\left(\mathbb{T}^{\nu}\right)^{2}$ и мероморфная по $z \in \mathbb{C}$, полюсы которой при любых $k$ u $p$ лежат вне множества значений фуункиии $\omega(k)$. При этом ядро $L(k, p ; z)$ симметрично и

$$
\int L(k, p ; z) d p=0 \text {. }
$$

Тогда ненулевое решение $f \in \bar{L}_{2}\left(\mathbb{T}^{\nu}\right)$ уравнения (2.2) существует для тех и только тех $z$, которые являются корнями уравнения $\Delta(z)=0$, где $\Delta(z)$ модифицированный детерминант Фредгольма,

$$
\begin{gathered}
\Delta(z)=\int_{\mathbb{T}^{\nu}} \frac{d k}{\omega(k)-z}+\sum_{n=2}^{\infty} \frac{1}{n !} \int_{\mathbb{T}^{\nu}} \ldots \int_{\mathbb{T}^{\nu}} \frac{T_{n}\left(k_{1}, \ldots, k_{n} ; z\right) d k_{1} \ldots d k_{n}}{\left(\omega\left(k_{1}\right)-z\right) \ldots\left(\omega\left(k_{n}\right)-z\right)}, \\
T_{n}\left(k_{1}, \ldots, k_{n} ; z\right)=\sum_{i=1}^{n} \operatorname{det}\left(\begin{array}{ccccc}
L\left(k_{1}, k_{1} ; z\right) & \ldots & 1 & \ldots & L\left(k_{1}, k_{n} ; z\right) \\
L\left(k_{2}, k_{1} ; z\right) & \ldots & 1 & \ldots & L\left(k_{2}, k_{n} ; z\right) \\
\vdots & & \vdots & & \vdots \\
L\left(k_{n}, k_{1} ; z\right) & \ldots & 1 & \ldots & L\left(k_{n}, k_{n} ; z\right)
\end{array}\right),
\end{gathered}
$$

где матрица в $i$-м слагаемом - это матрица $\left(L\left(k_{j}, k_{j^{\prime}} ; z\right), j, j^{\prime}=1, \ldots, n\right)$, в которой $i$-й столбеи, заменен на столбеи, состоящий из единич.

ДокАЗАтельство. Рассмотрим самосопряженный ограниченный оператор $\overline{\mathscr{T}}$ в пространстве $L_{2}\left(\mathbb{T}^{\nu}, d k\right)$, определенный формулой

$$
\begin{aligned}
(\overline{\mathscr{T}} f)(k)=\omega & (k) f(k)-\int_{\mathbb{T}^{\nu}} \omega(p) f(p) d p-\omega(k) \int_{\mathbb{T}^{\nu}} f(p) d p \\
& +\int_{\mathbb{T}^{\nu}} \omega(k) d k \int_{\mathbb{T}^{\nu}} f(p) d p+\int_{\mathbb{T}^{\nu}} L(k, p ; z) f(p) d p .
\end{aligned}
$$

Очевидно, что функция $f=$ const является собственной функцией этого оператора с собственным значением $z=0$, а в ортогональном дополнении $\bar{L}_{2}\left(\mathbb{T}^{\nu}, d k\right)$ $=(\text { const })^{\perp}$ собственные функции этого оператора с собственными значениями, лежащими вне отрезка $\left[\min \omega_{\Lambda}, \max \omega_{\Lambda}\right]$, удовлетворяют уравнению $(2.2)$ и, следовательно, являются нулями детерминанта Фредгольма $F(z)=1+$ $\sum_{n \geqslant 1} R_{n}(z)$, где

$$
R_{n}(z)=\frac{1}{n !} \int_{\mathbb{T}^{\nu}} \ldots \int_{\mathbb{T}^{\nu}} \frac{\mathscr{L}_{n}\left(k_{1}, \ldots, k_{n} ; z\right) d k_{1} \ldots d k_{n}}{\left(\omega\left(k_{1}\right)-z\right) \ldots\left(\omega\left(k_{n}\right)-z\right)},
$$

$\mathscr{L}_{n}\left(k_{1}, \ldots, k_{n}\right)=\operatorname{det}\left(L\left(k_{i}, k_{j} ; z\right)-\omega\left(k_{j}\right), i, j=1, \ldots, n\right)$.

Поскольку $j$-й столбец матрицы $\left(L\left(k_{i}, k_{j} ; z\right)-\omega\left(k_{j}\right), i, j=1, \ldots, n\right)$ представляется в виде суммы столбцов $\left(L\left(k_{i}, k_{j} ; z\right), i=1, \ldots, n\right)$ и $\left(\omega\left(k_{j}\right), \ldots, \omega\left(k_{j}\right)\right)$, то 
по свойству линейности определителя получаем

$$
\begin{aligned}
& \mathscr{L}_{n}\left(k_{1}, \ldots, k_{n}\right)=\operatorname{det}\left(\begin{array}{ccc}
L\left(k_{1}, k_{1} ; z\right) & \ldots & L\left(k_{1}, k_{n} ; z\right) \\
L\left(k_{2}, k_{1} ; z\right) & \ldots & L\left(k_{2}, k_{n} ; z\right) \\
\vdots & & \vdots \\
L\left(k_{n}, k_{1} ; z\right) & \ldots & L\left(k_{n}, k_{n} ; z\right)
\end{array}\right) \\
&-\sum_{i=1}^{n} \omega\left(k_{i}\right) \operatorname{det}\left(\begin{array}{ccccc}
L\left(k_{1}, k_{1} ; z\right) & \ldots & 1 & \ldots & L\left(k_{1}, k_{n} ; z\right) \\
L\left(k_{2}, k_{1} ; z\right) & \ldots & 1 & \ldots & L\left(k_{2}, k_{n} ; z\right) \\
\vdots & & \vdots & & \vdots \\
L\left(k_{n}, k_{1} ; z\right) & \ldots & 1 & \ldots & L\left(k_{n}, k_{n} ; z\right)
\end{array}\right) .
\end{aligned}
$$

(Определители матриц, в которых встречается больше одного столбца вида $\left(\omega\left(k_{j}\right), \ldots, \omega\left(k_{j}\right)\right)$, равны нулю.)

Для удобства положим

$\widehat{A}_{n}=\frac{1}{n !} \int_{\mathbb{T}^{\nu}} \ldots \int_{\mathbb{T}^{\nu}} \operatorname{det}\left(\begin{array}{ccc}L\left(k_{1}, k_{1} ; z\right) & \ldots & L\left(k_{1}, k_{n} ; z\right) \\ L\left(k_{2}, k_{1} ; z\right) & \ldots & L\left(k_{2}, k_{n} ; z\right) \\ \vdots & & \vdots \\ L\left(k_{n}, k_{1} ; z\right) & \ldots & L\left(k_{n}, k_{n} ; z\right)\end{array}\right) \frac{d k_{1} \ldots d k_{n}}{\prod_{i=1}^{n}\left(\omega\left(k_{i}\right)-z\right)}, \quad n \geqslant 1$.

$\widehat{A}_{0}=1$. Покажем, что

$$
R_{n}(z)=\widehat{A}_{n}-\widehat{A}_{n-1}-\frac{z}{n !} \int_{\mathbb{T}^{\nu}} \ldots \int_{\mathbb{T}^{\nu}} \frac{T_{n}\left(k_{1}, \ldots, k_{n} ; z\right) d k_{1} \ldots d k_{n}}{\left(\omega\left(k_{1}\right)-z\right) \ldots\left(\omega\left(k_{n}\right)-z\right)} .
$$

Воспользовавшись равенством

$$
\frac{\omega\left(k_{i}\right)}{\omega\left(k_{i}\right)-z}=1+\frac{z}{\omega\left(k_{i}\right)-z}
$$

мы получим, что

$$
\begin{aligned}
& \frac{1}{n !} \int_{\mathbb{T}^{\nu}} \ldots \int_{\mathbb{T}^{\nu}} \operatorname{det}\left(\begin{array}{ccccc}
L\left(k_{1}, k_{1} ; z\right) & \ldots & 1 & \ldots & L\left(k_{1}, k_{n} ; z\right) \\
L\left(k_{2}, k_{1} ; z\right) & \ldots & 1 & \ldots & L\left(k_{2}, k_{n} ; z\right) \\
\vdots & & \vdots & & \vdots \\
L\left(k_{n}, k_{1} ; z\right) & \ldots & 1 & \ldots & L\left(k_{n}, k_{n} ; z\right)
\end{array}\right) \frac{\omega\left(k_{i}\right) d k_{1} \ldots d k_{n}}{\prod_{i=1}^{n}\left(\omega\left(k_{i}\right)-z\right)} \\
& =\frac{\widehat{A}_{n-1}}{n}+\frac{z}{n !} \int_{\mathbb{T}^{\nu}} \ldots \int_{\mathbb{T}^{\nu}} \operatorname{det}\left(\begin{array}{ccccc}
L\left(k_{1}, k_{1} ; z\right) & \ldots & 1 & \ldots & L\left(k_{1}, k_{n} ; z\right) \\
L\left(k_{2}, k_{1} ; z\right) & \ldots & 1 & \ldots & L\left(k_{2}, k_{n} ; z\right) \\
\vdots & & \vdots & & \vdots \\
L\left(k_{n}, k_{1} ; z\right) & \ldots & 1 & \ldots & L\left(k_{n}, k_{n} ; z\right)
\end{array}\right) \frac{d k_{1} \ldots d k_{n}}{\prod_{i=1}^{n}\left(\omega\left(k_{i}\right)-z\right)} .
\end{aligned}
$$

При подстановке первого слагаемого из правой части формулы (2.8) в (2.9) возникает $\widehat{A}_{n-1} / n$. В самом деле, разложим определитель

$$
\operatorname{det}\left(\begin{array}{ccccc}
L\left(k_{1}, k_{1} ; z\right) & \ldots & 1 & \ldots & L\left(k_{1}, k_{n} ; z\right) \\
L\left(k_{2}, k_{1} ; z\right) & \ldots & 1 & \ldots & L\left(k_{2}, k_{n} ; z\right) \\
\vdots & & \vdots & & \vdots \\
L\left(k_{n}, k_{1} ; z\right) & \ldots & 1 & \ldots & L\left(k_{n}, k_{n} ; z\right)
\end{array}\right)
$$


по $i$-му столбцу (состоящему из единиц). Заметим, что при интегрировании мы получим ненулевой вклад только от минора, дополнительного к элементу, стоящему в позиции $(i, i)$ ( $i$-я строка, $i$-й столбец). В самом деле, знаменатель подынтегрального выражения в

$$
\int_{\mathbb{T}^{\nu}} \ldots \int_{\mathbb{T}^{\nu}} \operatorname{det}\left(\begin{array}{ccccc}
L\left(k_{1}, k_{1} ; z\right) & \ldots & 1 & \ldots & L\left(k_{1}, k_{n} ; z\right) \\
L\left(k_{2}, k_{1} ; z\right) & \ldots & 1 & \ldots & L\left(k_{2}, k_{n} ; z\right) \\
\vdots & & \vdots & & \vdots \\
L\left(k_{n}, k_{1} ; z\right) & \ldots & 1 & \ldots & L\left(k_{n}, k_{n} ; z\right)
\end{array}\right) \frac{d k_{1} \ldots d k_{n}}{\prod_{m=1, m \neq i}^{n}\left(\omega\left(k_{m}\right)-z\right)}
$$

не зависит от $k_{i}$, а минор, дополнительный к элементу, стоящему в позиции $(i, i)$, также не зависит от $k_{i}$ и равняется $\operatorname{det}\left(L\left(k_{i}, k_{j} ; z\right), i, j=1, \ldots, n-1\right)$. Остальные миноры представляются в виде суммы произведений $n-1$ мономов вида $\prod_{q \neq i} L\left(k_{p}, k_{q} ; z\right)$, причем в каждом из этих произведений есть и притом только один множитель, у которого $p=i$ и при этом $q \neq i$. Следовательно, по свойству (2.3), вклад при интегрировании от этих слагаемых будет нулевым.

Таким образом, равенство (2.9), а следовательно, и равенство (2.7) доказаны, и, значит,

$1+\sum_{n=1}^{N} \frac{1}{n !} R_{n}=\widehat{A}_{N}(z)-z\left[\sum_{n=2}^{N} \frac{1}{n !} \int_{\mathbb{T}^{\nu}} \ldots \int_{\mathbb{T}^{\nu}} \frac{T_{n}\left(k_{1}, \ldots, k_{n}\right)}{\prod_{i=1}^{n}\left(\omega\left(k_{i}\right)-z\right)} \prod_{i=1}^{n} d k_{i}+\int_{\mathbb{T}^{\nu}} \frac{d k}{\omega(k)-z}\right]$.

По известной лемме Адамара последовательность $\widehat{A}_{N}(z)$ стремится к нулю при $N \rightarrow \infty$ равномерно на любом компакте в $\Pi=\mathbb{C}^{1} \backslash[\min \omega(k), \max \omega(k)]$. Следовательно, детерминант Фредгольма равен $F(z)=-z \Delta(z)$.

Нулю функции $F(z)$ в точке $z=0$ соответствует собственная функция $f(k) \equiv$ const оператора $\overline{\mathscr{T}}$. Таким образом, нули функции $\Delta(z), z \in \Pi$, суть собственные значения оператора $\overline{\mathscr{T}}$ в подпространстве $\bar{L}_{2}\left(T^{\nu}, d k\right)$. Лемма 2.1 доказана.

Применительно к нашему случаю функция $T_{n}^{\Lambda}$, задаваемая формулой $(2.5)$, представляется в виде

$$
T_{n}^{\Lambda}\left(k_{1}, \ldots, k_{n}\right)=P_{n}^{\Lambda}\left(k_{1}, \ldots, k_{n}\right)-\frac{1}{\gamma(\Lambda)-z} Q_{n}^{\Lambda}\left(k_{1}, \ldots, k_{n}\right), \quad n \geqslant 2,
$$

где

$$
\begin{aligned}
& P_{n}^{\Lambda}\left(k_{1}, \ldots, k_{n}\right)=\sum_{i=1}^{n} \operatorname{det}\left(\begin{array}{ccccc}
K_{\Lambda}\left(k_{1}, k_{1}\right) & \ldots & 1 & \ldots & K_{\Lambda}\left(k_{1}, k_{n}\right) \\
K_{\Lambda}\left(k_{2}, k_{1}\right) & \ldots & 1 & \ldots & K_{\Lambda}\left(k_{2}, k_{n}\right) \\
\vdots & & \vdots & & \vdots \\
K_{\Lambda}\left(k_{n}, k_{1}\right) & \ldots & 1 & \ldots & K_{\Lambda}\left(k_{n}, k_{n}\right)
\end{array}\right) \\
& Q_{n}^{\Lambda}\left(k_{1}, \ldots, k_{n}\right)=\sum_{i \neq j}^{n} \bar{b}_{\Lambda}\left(k_{j}\right) \operatorname{det}\left(\begin{array}{ccccccc}
K_{\Lambda}\left(k_{1}, k_{1}\right) & \ldots & b_{\Lambda}\left(k_{1}\right) & \ldots & 1 & \ldots & K_{\Lambda}\left(k_{1}, k_{n}\right) \\
K_{\Lambda}\left(k_{2}, k_{1}\right) & \ldots & b_{\Lambda}\left(k_{2}\right) & \ldots & 1 & \ldots & K_{\Lambda}\left(k_{2}, k_{n}\right) \\
\vdots & & \vdots & & \vdots & & \vdots \\
K_{\Lambda}\left(k_{n}, k_{1}\right) & \ldots & b_{\Lambda}\left(k_{n}\right) & \ldots & 1 & \ldots & K_{\Lambda}\left(k_{n}, k_{n}\right)
\end{array}\right)
\end{aligned}
$$


Введем величины

$$
\begin{aligned}
& I_{n}^{1, \Lambda}(z)=\frac{1}{n !} \int_{\mathbb{T}^{\nu}} \ldots \int_{\mathbb{T}^{\nu}} \frac{P_{n}^{\Lambda}\left(k_{1}, \ldots, k_{n}\right)}{\prod_{i=1}^{n}\left(\omega_{\Lambda}\left(k_{i}\right)-z\right)} \prod_{i=1}^{n} d k_{i}, \quad n \geqslant 2, \\
& I_{n}^{2, \Lambda}(z)=\frac{1}{n !} \int_{\mathbb{T}^{\nu}} \ldots \int_{\mathbb{T}^{\nu}} \frac{Q_{n}^{\Lambda}\left(k_{1}, \ldots, k_{n}\right)}{\prod_{i=1}^{n}\left(\omega_{\Lambda}\left(k_{i}\right)-z\right)} \prod_{i=1}^{n} d k_{i}, \quad n \geqslant 2 .
\end{aligned}
$$

Из представлений (1.16), формулы Стирлинга и леммы Адамара нетрудно получить следующие оценки:

$$
\begin{aligned}
& \left|I_{n}^{1, \Lambda}(z)\right|<A \frac{\left(C \beta^{2}\right)^{n-1}}{n^{(n-1) / 2} d^{n-1}} \int_{\mathbb{T}^{\nu}} \frac{d k}{\left|\omega_{\Lambda}(k)-z\right|}, \\
& \left|I_{n}^{2, \Lambda}(z)\right|<A \frac{\left(C \beta^{2}\right)^{n-2}}{n^{(n-2) / 2} d^{n-2}} \int_{\mathbb{T}^{\nu}} \int_{\mathbb{T}^{\nu}} \frac{\left|b_{\Lambda}\left(k_{1}\right)-b_{\Lambda}\left(k_{2}\right)\right|^{2} d k_{1} d k_{2}}{\left|\omega_{\Lambda}\left(k_{1}\right)-z\right|\left|\omega_{\Lambda}\left(k_{2}\right)-z\right|},
\end{aligned}
$$

где $C$ и $A-$ некоторые константы, a $d=\operatorname{dist}\left(z, \Pi_{\Lambda}^{\prime}\right)-$ расстояние от точки $z \in \mathbb{C}^{1}$ до $\Pi_{\Lambda}^{\prime}=\left(\tau_{\Lambda}^{\min }, \tau_{\Lambda}^{\max }\right)-$ множества значений функции $\omega_{\Lambda}$.

Далее, в силу (2.10) ряды

$$
\begin{aligned}
& R_{1}^{\Lambda}(z)=\sum_{n=2}^{\infty} I_{n}^{1, \Lambda}(z), \\
& R_{2}^{\Lambda}(z)=\sum_{n=2}^{\infty} I_{n}^{2, \Lambda}(z)=\int_{\mathbb{T}^{\nu}} \int_{\mathbb{T}^{\nu}} \frac{\left|b_{\Lambda}\left(k_{1}\right)-b_{\Lambda}\left(k_{2}\right)\right|^{2} d k_{1} d k_{2}}{\left(\omega_{\Lambda}\left(k_{1}\right)-z\right)\left(\omega_{\Lambda}\left(k_{2}\right)-z\right)}+\bar{R}_{2}^{\Lambda}(z)
\end{aligned}
$$

оцениваются степенными рядами по переменной $C \beta^{2} / d$, представляющими целые функции второго порядка от этой переменной. Из известных формул теории целых функций (см. [14]) следуют оценки

$$
\begin{aligned}
& \left|R_{1}^{\Lambda}(z)\right|<\overline{\bar{A}}_{1} \frac{C \beta^{2}}{d^{2}} e^{M_{1}\left(C \beta^{2} / d\right)^{2}} \int_{\mathbb{T}^{\nu}} \frac{d k}{\left|\omega_{\Lambda}(k)-z\right|}, \\
& \left|R_{2}^{\Lambda}(z)\right|<\overline{\bar{A}}_{2} \frac{C \beta^{2}}{d^{2}} e^{M_{2}\left(C \beta^{2} / d\right)^{2}} \int_{\mathbb{T}^{\nu}} \int_{\mathbb{T}^{\nu}} \frac{\left|b_{\Lambda}\left(k_{1}\right)-b_{\Lambda}\left(k_{2}\right)\right|^{2} d k_{1} d k_{2}}{\left|\omega_{\Lambda}\left(k_{1}\right)-z\right|\left|\omega_{\Lambda}\left(k_{2}\right)-z\right|},
\end{aligned}
$$

где $\overline{\bar{A}}_{1}, \overline{\bar{A}}_{2}, M_{1}, M_{2}$ - константы.

Для большей определенности будем считать, что $\gamma_{0}<\omega_{0}$, т. е. значения функции $\gamma(\Lambda)$ лежат левее множества $\Pi_{\Lambda}^{\prime}$. Случай $\gamma_{0}>\omega_{0}$ разбирается аналогично.

Далее мы будем последовательно рассматривать следующие интервалы вещественной оси:

$$
\begin{array}{cl}
\text { I. } & -\infty<z \leqslant \gamma(\Lambda)-D_{1} \beta^{2}, \\
\text { II. } & \gamma(\Lambda)-D_{1} \beta^{2}<z<\gamma(\Lambda), \\
\text { III. } & \gamma(\Lambda)<z<\tau_{\Lambda}^{\min }-D_{2} \beta^{2}, \\
\text { IV } . & \tau_{\Lambda}^{\max }+D_{2} \beta^{2}<z<\infty,
\end{array}
$$

где константы $D_{1}$ и $D_{2}$ выбираются так, что

$$
\overline{\bar{A}}_{i} \frac{C}{D_{i}} e^{M_{i} C / D_{i}}<\frac{1}{2}, \quad i=1,2 .
$$


При этом верны следующие неравенства:

$$
\begin{gathered}
\int_{\mathbb{T}^{\nu}} \frac{d k}{\omega_{\Lambda}(k)-z}+R_{1}^{\Lambda}(z)>\frac{1}{2} \int_{\mathbb{T}^{\nu}} \frac{d k}{\omega_{\Lambda}(k)-z}>0 \quad \text { при } z<\tau_{\Lambda}^{\min }-D_{2} \beta^{2}, \\
\int_{\mathbb{T}^{\nu}} \frac{d k}{\omega_{\Lambda}(k)-z}+R_{1}^{\Lambda}(z)<\frac{1}{2} \int_{\mathbb{T}^{\nu}} \frac{d k}{\omega_{\Lambda}(k)-z}<0 \quad \text { при } z>\tau_{\Lambda}^{\max }+D_{2} \beta^{2} .
\end{gathered}
$$

Далее,

$$
\begin{aligned}
0 & <\frac{1}{2} \int_{\mathbb{T}^{\nu}} \int_{\mathbb{T}^{\nu}} \frac{\left|b_{\Lambda}\left(k_{1}\right)-b_{\Lambda}\left(k_{2}\right)\right|^{2} d k_{1} d k_{2}}{\left(\omega_{\Lambda}\left(k_{1}\right)-z\right)\left(\omega_{\Lambda}\left(k_{2}\right)-z\right)}<R_{2}^{\Lambda}(z) \\
& <\frac{3}{2} \int_{\mathbb{T}^{\nu}} \int_{\mathbb{T}^{\nu}} \frac{\left|b_{\Lambda}\left(k_{1}\right)-b_{\Lambda}\left(k_{2}\right)\right|^{2} d k_{1} d k_{2}}{\left(\omega_{\Lambda}\left(k_{1}\right)-z\right)\left(\omega_{\Lambda}\left(k_{2}\right)-z\right)}<\frac{3}{2} \frac{C}{D_{2}} \int_{\mathbb{T}^{\nu}} \frac{d k}{\left|\omega_{\Lambda}(k)-z\right|} \quad \text { при } d>D_{2} \beta^{2},
\end{aligned}
$$

где $C>0-$ некоторая константа, не зависящая от $z$ и $\Lambda$.

Из этих неравенств следует, что

$$
\Delta_{\Lambda}(z)=\frac{d k}{\omega_{\Lambda}(k)-z}+R_{1}^{\Lambda}(z)-\frac{1}{\gamma(\Lambda)-z} R_{2}^{\Lambda}(z)>0
$$

на интервале III и при достаточно больших $D_{2}$ выполнено неравенство $\Delta_{\Lambda}(z)<0$ на интервале IV.

Наконец, на интервале I

$$
R_{2}^{\Lambda}(z)<\frac{\bar{C} \beta^{2}}{\tau_{\Lambda}^{\min }-\gamma(\Lambda)} \int_{\mathbb{T}^{\nu}} \frac{d k}{\omega_{\Lambda}(k)-z}
$$

где $\bar{C}$ - константа и, следовательно, на этом интервале

$$
\left|\frac{1}{\gamma(\Lambda)-z} R_{2}^{\Lambda}(z)\right|<\frac{\bar{C}}{D_{1}} \int_{\mathbb{T}^{\nu}} \frac{d k}{\omega_{\Lambda}(k)-z}<\frac{1}{3} \int_{\mathbb{T}^{\nu}} \frac{d k}{\omega_{\Lambda}(k)-z},
$$

если подходящим образом выбрать $D_{1}$. Отсюда и из предыдущих неравенств мы получаем, что на интервале I выполнено неравенство $\Delta_{\Lambda}(z)>0$.

Таким образом, нам остается изучить $\Delta_{\Lambda}(z)$ на интервале II. Поскольку на правом конце этого интервала $\Delta_{\Lambda}(z)$ принимает значение $-\infty$ (в силу положительности $R_{2}^{\Lambda}(z)$ ), а на левом положительна, существует по крайней мере один нуль этой функции. Для того чтобы показать, что этот нуль единственный, мы докажем монотонность этой функции на интервале II. Имеем

$$
\Delta_{\Lambda}^{\prime}(z)=\int_{\mathbb{T}^{\nu}} \frac{d k}{\left(\omega_{\Lambda}(k)-z\right)^{2}}+\left(R_{1}^{\Lambda}\right)^{\prime}(z)-\frac{1}{(\gamma(\Lambda)-z)^{2}} R_{2}^{\Lambda}(z)-\frac{1}{\gamma(\Lambda)-z}\left(R_{2}^{\Lambda}\right)^{\prime}(z) .
$$

Поскольку

$$
\left(I_{n}^{1, \Lambda}(z)\right)^{\prime}=\sum_{i=1}^{n} \int_{\mathbb{T}^{\nu}} \ldots \int_{\mathbb{T}^{\nu}} \frac{P_{n}^{\Lambda}\left(k_{1}, \ldots, k_{n}\right) d k_{1} \ldots d k_{n}}{\left(\omega_{\Lambda}\left(k_{i}\right)-z\right)^{2} \prod_{j \neq i}\left(\omega_{\Lambda}\left(k_{j}\right)-z\right)}
$$

и верна аналогичная формула для $\left(I_{n}^{2, \Lambda}(z)\right)^{\prime}$, мы, повторяя предыдущие рассуждения, получим, что на интервале II

$$
\left|\int_{\mathbb{T}^{\nu}} \frac{d k}{\left(\omega_{\Lambda}(k)-z\right)^{2}}+R_{1}^{\Lambda^{\prime}}(z)\right|<\text { const }_{1}, \quad\left|R_{2}^{\Lambda^{\prime}}(z)\right|<\text { const }_{2} \beta^{2},
$$

где const $_{1}$, const $_{2}$ не зависят от $z, \Lambda, \beta$. 
Далее, поскольку на интервале II (в силу условий (1.17))

$$
\int_{\mathbb{T}^{\nu}} \int_{\mathbb{T}^{\nu}} \frac{\left|b_{\Lambda}\left(k_{1}\right)-b_{\Lambda}\left(k_{2}\right)\right|^{2} d k_{1} d k_{2}}{\left(\omega_{\Lambda}\left(k_{1}\right)-z\right)\left(\omega_{\Lambda}\left(k_{2}\right)-z\right)}>\text { const } \int_{\mathbb{T}^{\nu}}\left|b_{\Lambda}(k)\right|^{2} d k>\text { const }_{3} \beta^{2},
$$

мы получаем, что на этом интервале

$$
\Delta_{\Lambda}^{\prime}(z)<\text { const }_{1}-\frac{\text { const }_{2} \beta^{2}}{(\gamma(\Lambda)-z)^{2}}<\text { const }_{1}-\frac{\text { const }_{2}}{D_{1}^{2} \beta^{2}}<0
$$

при достаточно малых $\beta$. Здесь const $_{1}$, const $_{2}$ не зависят от $\beta$ и $\Lambda$.

Таким образом, мы доказали существование единственного нуля $\varepsilon_{b}=\varepsilon_{b}(\Lambda)$ функции $\Delta_{\Lambda}(z)$ вне $D_{2} \beta^{2}$-окрестности непрерывного спектра. Этот нуль находится от $\gamma(\Lambda)$ на расстоянии, не превосходящем $D_{1} \beta^{2}$. Поскольку $\gamma(\Lambda)-\gamma_{0}=$ $O\left(\beta^{2}\right)$, утверждение теоремы 2.1 доказано.

\section{§3. Отсутствие прилегающих уровней вдали от особых значений $\Lambda$}

Далее следует изучить собственные значения $\mathscr{T}_{\Lambda}$, лежащие в $D_{2} \beta^{2}$-окрестности непрерывного спектра - так называемые прилегающие уровни. Они возникают лишь вблизи особых значений $\Lambda$ : это те значения, в которых функция $\omega_{\Lambda}(k)$ имеет либо вырожденный экстремум (глобальный), либо кратные экстремумы. Здесь мы покажем, что при $\Lambda$, лежащих вдали от особых значений, у оператора $\mathscr{T}_{\Lambda}$ нет прилегающих уровней.

Tеорема 3.1. Пусть $V_{\delta} \subset \mathbb{T}^{\nu}$ есть $\delta$-окрестность множества особых значений $\Lambda, \delta>0$. Тогда существует такое $\beta_{0}=\beta_{0}(\delta)$, что при $|\beta|<\beta_{0}$ и любом $\Lambda \notin V_{\delta}$ у оператора $\mathscr{T}_{\Lambda}$ нет прилегающих уровней.

ДокАЗАТЕльСтво. Ради простоты изложения мы проведем здесь доказательство лишь для случая $\nu=1$. Оно обобщается на случай более высоких размерностей $\nu$.

В случае, когда $\Lambda \notin V_{\delta}$, имеется единственная точка $k_{\Lambda}^{\max }$, в которой функция $\omega_{\Lambda}(k)$ достигает максимума и этот экстремум невырожден, и аналогичная точка $k_{\Lambda}^{\min }$, где она достигает минимума. У этих точек существуют $\varepsilon$-окрестности $U_{\varepsilon}^{\max }$ и $U_{\varepsilon}^{\min }$ соответственно, внутри которых производная $\omega_{\Lambda}^{\prime \prime}(k)$ не меняет свой знак, а вне окрестностей $U_{\varepsilon}^{\mathrm{ext}}$, ext $=\min , \max$, выполнены неравенства

$$
\begin{array}{ll}
\omega_{\Lambda}(k)-\omega_{\Lambda}\left(k_{\Lambda}^{\min }\right)>\beta \rho, & k \notin U_{\varepsilon}^{\min }, \\
\omega_{\Lambda}\left(k_{\Lambda}^{\max }\right)-\omega_{\Lambda}(k)>\beta \rho, & k \notin U_{\varepsilon}^{\max },
\end{array}
$$

где $\rho>0$ - некоторая константа, зависящая от $\beta$ и $\Lambda$. В дальнейшем, не умаляя общности, будем считать, что $\varepsilon<1 / 3$.

Лемма 3.1. Для $\Lambda \notin V_{\delta} u z<\tau_{\Lambda}^{\min }$ верно следующее представление:

$$
\int_{\mathbb{T}^{1}} \frac{d k}{\omega_{\Lambda}(k)-z}=\frac{A_{\min }^{\Lambda}}{\sqrt{\tau_{\Lambda}^{\min }-z}}+\psi_{\Lambda}^{\min }(z)
$$

где $A_{\min }^{\Lambda}=\sqrt{2} \pi\left|\omega_{\Lambda}^{\prime \prime}\left(k_{\Lambda}^{\min }\right)\right|^{-1 / 2}, \tau_{\Lambda}^{\min }=\omega_{\Lambda}\left(k_{\Lambda}^{\min }\right)$, a $\psi_{\Lambda}^{\min }(z)-$ ограниченнал функиия от $z,-\infty<z<\tau_{\Lambda}^{\min }$, допускающал оченку $\left|\psi_{\Lambda}^{\min }(z)\right| \leqslant C / \beta$, где константа $C$ не зависит от $\beta$ и $\Lambda$. При $z>\tau_{\Lambda}^{\max }$ имеет место аналогичное представление. 
ДокАЗАТЕЛЬСтво. Запишем

$$
\int_{\mathbb{T}^{1}} \frac{d k}{\omega_{\Lambda}(k)-z}=\int_{U_{\varepsilon}^{\min }} \frac{d k}{\omega_{\Lambda}(k)-z}+\int_{\mathbb{T}^{1} \backslash U_{\varepsilon}^{\min }} \frac{d k}{\omega_{\Lambda}(k)-z} .
$$

В силу оценки (3.1) второй интеграл не превосходит $2 \pi /(\beta \rho)$. Для оценки первого интеграла совершим гладкую замену переменных $k \rightarrow x(k)$ в окрестности $U_{\varepsilon}^{\min }$

$$
k=k_{\Lambda}^{\min }+x+a x^{2}+O\left(x^{3}\right), \quad-\varepsilon_{1}^{\prime}<x<\varepsilon_{2}^{\prime},
$$

где $a$ - некоторая константа, а $\varepsilon_{i}^{\prime}=\varepsilon_{i}^{\prime}(\varepsilon)=O(\varepsilon)$, приводящую функцию $\omega_{\Lambda}(k)$ к виду

$$
\omega_{\Lambda}(k)=\tau_{\Lambda}^{\min }+\frac{1}{2} \omega_{\Lambda}^{\prime \prime}\left(k_{\Lambda}^{\min }\right) x^{2}
$$

Первый интеграл в (3.3) представится в виде

$$
\begin{aligned}
\int_{-\varepsilon_{1}^{\prime}}^{\varepsilon_{2}^{\prime}} & \frac{C(x) d x}{\frac{1}{2} \omega_{\Lambda}^{\prime \prime}\left(k_{\Lambda}^{\text {min }}\right) x^{2}+\tau_{\Lambda}^{\min }-z}=\int_{-\varepsilon_{1}^{\prime}}^{\varepsilon_{2}^{\prime}} \frac{d x}{\frac{1}{2} \omega_{\Lambda}^{\prime \prime}\left(k_{\Lambda}^{\text {min }}\right) x^{2}+\tau_{\Lambda}^{\text {min }}-z} \\
& +2 a \int_{-\varepsilon_{1}^{\prime}}^{\varepsilon_{2}^{\prime}} \frac{x d x}{\frac{1}{2} \omega_{\Lambda}^{\prime \prime}\left(k_{\Lambda}^{\min }\right) x^{2}+\tau_{\Lambda}^{\min }-z}+\int_{-\varepsilon_{1}^{\prime}}^{\varepsilon_{2}^{\prime}} \frac{x^{2} \widetilde{C}(x) d x}{\frac{1}{2} \omega_{\Lambda}^{\prime \prime}\left(k_{\Lambda}^{\text {min }}\right) x^{2}+\tau_{\Lambda}^{\min }-z}
\end{aligned}
$$

здесь $C(x)=d k / d x=1+2 a x+x^{2} \widetilde{C}(x)$, где $\widetilde{C}(x)$ - ограниченная функция. Второй и третий интегралы в (3.4) снова допускают оценку $\bar{c} / \beta$ ( $\bar{c}$ не зависит от $\beta$ и $\Lambda)$, а первый представляется в виде

$$
\begin{aligned}
\int_{-\varepsilon_{1}^{\prime}}^{\varepsilon_{2}^{\prime}} & \frac{d x}{\frac{1}{2} \omega_{\Lambda}^{\prime \prime}\left(k_{\Lambda}^{\text {min }}\right) x^{2}+\tau_{\Lambda}^{\text {min }}-z} \\
& =\int_{-\infty}^{\infty} \frac{d x}{\frac{1}{2} \omega_{\Lambda}^{\prime \prime}\left(k_{\Lambda}^{\text {min }}\right) x^{2}+\tau_{\Lambda}^{\text {min }}-z}-\int_{\mathbb{R}^{1} \backslash\left[-\varepsilon_{1}^{\prime}, \varepsilon_{2}^{\prime}\right]} \frac{d x}{\frac{1}{2} \omega_{\Lambda}^{\prime \prime}\left(k_{\Lambda}^{\text {min }}\right) x^{2}+\tau_{\Lambda}^{\text {min }}-z} .
\end{aligned}
$$

Второй интеграл снова может быть оценен величиной $\bar{c} / \beta$, а первый равен первому слагаемому в (3.2). Лемма доказана.

Перейдем теперь к оценке ряда

$$
\sum_{n \geqslant 2} \frac{1}{n !} I_{n}^{\Lambda}(z)=R_{1}^{\Lambda}(z)-\frac{1}{\gamma(\Lambda)-z} R_{2}^{\Lambda}(z)
$$

при $z<\tau_{\Lambda}^{\min }$. Здесь

$$
I_{n}^{\Lambda}(z)=\int_{\mathbb{T}^{1}} \ldots \int_{\mathbb{T}^{1}} \frac{T_{n}^{\Lambda}\left(k_{1}, \ldots, k_{n}\right)}{\prod_{i=1}^{n}\left(\omega_{\Lambda}\left(k_{i}\right)-z\right)} \prod_{i=1}^{n} d k_{i}, \quad n \geqslant 2,
$$

a $T_{k}^{\Lambda}$ определена формулой $(2.10)$.

Мы воспользуемся следующей оценкой:

Лемма 3.2. Для $z$, лежащих в $D_{2} \beta^{2}$-окрестности одного из краев $\tau_{\Lambda}^{\text {min }}$ или $\tau_{\Lambda}^{\max }$ непрерьвного спектра, и любого из подмножеств индексов $\gamma \subset\{1, \ldots, n\}$, $\# \gamma \geqslant 2$, верна оченка

$$
\left|T_{n}^{\Lambda}\left(k_{1}, \ldots, k_{n}, z\right)\right|<A\left(C \beta^{2}\right)^{n-1} n^{n / 2} \prod_{(i, j) \subseteq \gamma}\left(k_{i}-k_{j}\right)^{2},
$$


где $A$ и $\mathrm{C}$ константы, не зависящие от $\gamma, \beta, \Lambda, k_{i}, i=1, \ldots, n, u z, a$ произведение берется по всем неупорядоченным парам $(i, j)$ элементов из $\gamma$.

Доказательство этой леммы приводится в приложении.

ЛЕмма 3.3. При $z<\tau_{\Lambda}^{\min }$ верна оценка

$$
\left|\sum_{n=2}^{\infty} I_{n}^{\Lambda}(z)\right| \leqslant B \frac{A_{\min }^{\Lambda} \beta}{\sqrt{\tau_{\Lambda}^{\min }-z}}+R
$$

где $R, B$ - константы, не зависящие от $\beta, z и \Lambda$.

ДокАЗАТЕЛЬСТво. Положим для каждого $\gamma \subset\{1, \ldots, n\}$

$$
\mathscr{H}_{\gamma}=\left\{\left(k_{1}, \ldots, k_{n}\right) \in\left(\mathbb{T}^{1}\right)^{n}: k_{i} \in U_{\varepsilon}^{\min }, i \in \gamma, k_{j} \notin U_{\varepsilon}^{\min }, j \notin \gamma\right\},
$$

где $U_{\varepsilon}^{\min }$ есть $\varepsilon$-окрестность точки $k_{\Lambda}^{\min }$.

Тогда при $\# \gamma \geqslant 2$

$\left|\int_{\mathscr{H}_{\gamma}} \frac{T_{n}^{\Lambda}\left(k_{1}, \ldots, k_{n}\right)}{\prod_{i=1}^{n}\left(\omega_{\Lambda}\left(k_{i}\right)-z\right)} \prod_{i=1}^{n} d k_{i}\right|<\frac{A\left(C \beta^{2}\right)^{n-1} n^{n / 2}}{(\beta \rho)^{n-|\gamma|}} \int_{\mathscr{H}_{\gamma}} \frac{\prod_{(i, j) \subseteq \gamma}\left(k_{i}-k_{j}\right)^{2}}{\prod_{i \in \gamma}\left(\omega_{\Lambda}\left(k_{i}\right)-z\right)} \prod_{i \in \gamma} d k_{i}$.

Далее, положив $u_{i}=k_{i}-k_{\Lambda}^{\min }, i \in \gamma$, получим, что

$$
\prod_{(i, j) \subset \gamma}\left(k_{i}-k_{j}\right)^{2} \leqslant \prod_{(i, j) \subset \gamma} 2\left(u_{i}^{2}+u_{j}^{2}\right)=\sum_{s=\left\{s_{i}, i \in \gamma\right\}} A_{s} \prod\left(2 u_{i}^{2}\right)^{s_{i}},
$$

где суммирование происходит по всем мультииндексам $s$ с некоторыми комбинаторными положительными коэффициентами $A_{s}$, сумма которых равна $2^{p(p-1) / 2}$, $p=|\gamma|$. Заметим, что в каждом мультииндексе $s=\left\{s_{i}, i \in \gamma\right\}$ существует не более одного значения $s_{i}=0$; поэтому сумму (3.10) можно записать в виде

$$
\sum_{j \in \gamma} \prod_{i \neq j, i \in \gamma}\left(2 u_{i}^{2}\right) \sum_{s: s_{j}=0} A_{s} \prod_{m \neq j, m \in \gamma}\left(2 u_{m}^{2}\right)^{s_{m}-1}+\prod_{i \in \gamma}\left(2 u_{i}\right)^{2} \sum_{s: s_{i}>0} A_{s} \prod_{m \in \gamma}\left(2 u_{m}\right)^{s_{m}-1} .
$$

Далее, поскольку $\left|u_{i}\right|<\varepsilon$ и $\sum s_{i}=p(p-1) / 2$, мы получаем, что

$$
\sum_{s: s_{j}=0} A_{s} \prod\left(2 u_{m}^{2}\right)^{s_{m}-1}<2^{p-1}\left(4 \varepsilon^{2}\right)^{(p-1)(p-2) / 2}<2^{p-1}
$$

и

$$
\sum_{s, s_{i}>0} A_{s} \prod\left(2 u_{m}\right)^{s_{m}}<\left(4 \varepsilon^{2}\right)^{p(p-1) / 2}<1
$$

при $4 \varepsilon^{2}<1$. Таким образом, интеграл в (3.9) допускает оценку

$$
\begin{aligned}
& \int_{\mathscr{H}_{\gamma}} \frac{\prod\left(k_{i}-k_{j}\right)^{2}}{\prod\left(\omega_{\Lambda}\left(k_{i}\right)-z\right)} \prod d k_{i} \\
& \quad<2^{p-1} \sum_{j \in \gamma} \int_{U_{\varepsilon}^{\min }} \frac{d k_{j}}{\omega_{\Lambda}\left(k_{j}\right)-z} \prod_{i \neq j, i \in \gamma} \int_{U_{\varepsilon}^{\min }} \frac{2 u_{i}^{2} d k_{i}}{\omega_{\Lambda}\left(k_{i}\right)-z}+\prod_{i \in \gamma} \int_{U_{\varepsilon}^{\min }} \frac{2 u_{i}^{2} d k}{\omega_{\Lambda}\left(k_{i}\right)-z} \\
& \quad \leqslant\left(\frac{\bar{C} \varepsilon}{\beta}\right)^{p-1} p \int_{U_{\varepsilon}^{\min }} \frac{d k}{\omega_{\Lambda}(k)-z}+\left(\frac{2 \bar{C} \varepsilon}{\beta}\right)^{p}
\end{aligned}
$$

где $\bar{C}$ - некоторая константа, не зависящая от $\beta, \Lambda, z$. 
Окончательная оценка для интеграла (3.9) имеет вид

$$
\begin{aligned}
& \left|\int_{\mathscr{H}_{\gamma}} \frac{T_{n}^{\Lambda}\left(k_{1}, \ldots, k_{n}\right)}{\prod_{i=1}^{n}\left(\omega_{\Lambda}\left(k_{i}\right)-z\right)} \prod_{i=1}^{n} d k_{i}\right| \\
& \quad<A\left(C \beta^{2}\right)^{n-1} n^{n / 2}\left[\left(\frac{D}{\beta}\right)^{n-1}|\gamma| \int_{U_{\varepsilon}^{\min }} \frac{d k}{\omega_{\Lambda}(k)-z}+\left(\frac{D}{\beta}\right)^{n}\right] \\
& =A(\widehat{C} \beta)^{n-1} n^{n / 2}\left[|\gamma| \int_{U_{\varepsilon}} \frac{d k}{\omega_{\Lambda}(k)-z}+\frac{D}{\beta}\right],
\end{aligned}
$$

где $\widehat{C}$ и $D-$ константы.

Таким образом, суммируя по всем $\gamma$, получаем, что

$$
\left|\int_{\left(\mathbb{T}^{1}\right)^{n}} \frac{T_{n}^{\Lambda}\left(k_{1}, \ldots, k_{n} ; z\right)}{\prod_{i=1}^{n}\left(\omega_{\Lambda}\left(k_{i}\right)-z\right)} \prod_{i=1}^{n} d k_{i}\right|<\widehat{A}(\widehat{\widehat{C}} \beta)^{n-1} n^{n / 2+1}\left[\int_{\mathbb{T}^{\nu}} \frac{d k}{\omega_{\Lambda}(k)-z}+\frac{\bar{D}}{\beta}\right],
$$

где снова $\bar{A}, \widehat{\widehat{C}}, \bar{D}$ - константы, не зависящие от $\beta, \Lambda, n$ и $z$.

Отсюда, наконец, следует, что ряд (3.5) не превосходит $B A_{\min }^{\Lambda} \beta / \sqrt{\tau_{\Lambda}^{\text {min }}-z}+R$, где $B$ и $R$ - константы, не зависящие от $\beta, z$ и $\Lambda$. В выводе последнего неравенства мы использовали рассуждения из доказательства леммы 3.1. Лемма 3.3 доказана.

В силу предыдущих оценок при $\tau_{\Lambda}^{\min }-D_{2} \beta^{2}<z<\tau_{\Lambda}^{\min }$ функция $\Delta_{\Lambda}(z)$ представляется при достаточно малом $\beta$ в виде

$$
\Delta_{\Lambda}(z)=\frac{\varphi_{\Lambda}(z)}{\sqrt{\tau_{\Lambda}^{\text {min }}-z}}+\widehat{\psi}_{\Lambda}(z)
$$

где $\left|\varphi_{\Lambda}(z)-A_{\min }^{\Lambda}\right|<B \beta$ и $\left|\widehat{\psi}_{\Lambda}(z)\right|<\hat{c} / \beta$.

Поскольку $A_{\Lambda}^{\text {min }} \sim 1 / \sqrt{\beta}$, a $\sqrt{\tau_{\Lambda}^{\text {min }}-z}<\sqrt{D_{2}} \beta$, мы видим, что первый член в представлении (3.13) при малых $\beta$ превосходит по абсолютной величине второй, и, следовательно, $\Delta_{\Lambda}(z) \neq 0$ в указанном диапазоне $z$. Таким образом, доказано, что при $\Lambda \notin V_{\delta}$ у оператора $\mathscr{T}_{\Lambda}$ нет прилегающих уровней.

Теорема 3.1 доказана.

\section{§4. Приложение. Доказательство леммы 3.2}

Рассмотрим детерминант

$$
\operatorname{det}\left(L\left(k_{i}, q_{j}\right)\right)=\operatorname{det}\left(\begin{array}{ccc}
L\left(k_{1}, q_{1}\right) & \ldots & L\left(k_{1}, q_{n}\right) \\
L\left(k_{2}, q_{1}\right) & \ldots & L\left(k_{2}, q_{n}\right) \\
\vdots & & \vdots \\
L\left(k_{n}, q_{1}\right) & \ldots & L\left(k_{n}, q_{n}\right)
\end{array}\right)
$$

где $L(k, q)$ - функция двух переменных $(k, q) \in \mathbb{T}^{1} \times \mathbb{T}^{1}$, аналитическая по каждому переменному в достаточно большой комплексной окрестности $W_{A}$ вида (1.4), где $A>1$. В этом случае из формулы Коши легко следует, что

$$
\left|L^{\left(s_{1}\right)\left(s_{2}\right)}(k, q)\right|<D s_{1} ! s_{2} !,
$$

где $D=\max _{k \in W_{A}, q \in W_{A}}|L(k, q)|$, а $L^{\left(s_{1}\right)\left(s_{2}\right)}$ означает производную порядка $s_{1}$ по первому и $s_{2}$ по второму аргументам. 
Без ограничения общности мы можем считать, что подмножество $\gamma$ состоит из первых $p$ элементов множества $\{1, \ldots, n\}$. Верна следующая легко проверяемая формула:

$$
\operatorname{det}\left(L\left(k_{i}, q_{j}\right)\right)=\int_{k_{1}}^{k_{2}} d \xi_{2} \int_{k_{1}}^{k_{3}} d \xi_{3} \ldots \int_{k_{1}}^{k_{p}} d \xi_{p}\left|\begin{array}{ccc}
L\left(k_{1}, q_{1}\right) & \ldots & L\left(k_{1}, q_{n}\right) \\
L^{(1)(0)}\left(\xi_{2}, q_{1}\right) & \ldots & L^{(1)(0)}\left(\xi_{2}, q_{n}\right) \\
\vdots & & \vdots \\
L^{(1)(0)}\left(\xi_{p}, q_{1}\right) & \ldots & L^{(1)(0)}\left(\xi_{p}, q_{n}\right) \\
L\left(k_{p+1}, q_{1}\right) & \ldots & L\left(k_{p+1}, q_{n}\right) \\
L\left(k_{n}, q_{1}\right) & \ldots & L\left(k_{n}, q_{n}\right)
\end{array}\right| .
$$

Применяя этот прием последовательно к переменным $\xi_{2}, \ldots, \xi_{p}$ и используя затем аналогичное представление для переменных $q_{1}, \ldots, q_{n}$, получаем окончательно

$$
\begin{aligned}
& \operatorname{det}\left(L\left(k_{i}, q_{j}\right)\right) \\
& =\int_{k_{1}}^{k_{2}} d \xi_{2}^{(1)} \ldots \int_{k_{1}}^{k_{p}} d \xi_{p}^{(1)} \int_{\xi_{2}^{(1)}}^{\xi_{3}^{(1)}} d \xi_{3}^{(2)} \ldots \int_{\xi_{2}^{(1)}}^{\xi_{p}^{(1)}} d \xi_{p}^{(2)} \ldots \int_{\xi_{p-2}^{(p-3)}}^{\xi_{p}^{(p-3)}} d \xi_{\bar{p}}^{(p-2)} \int_{\xi_{p-2}^{(p-3)}}^{\xi_{p}^{(p-3)}} d \xi_{p}^{(p-2)} \int_{\xi_{p-1}^{(p-2)}}^{\xi_{p}^{(p-2)}} d \xi_{p}^{(p-1)} \\
& \times \int_{q_{1}}^{q_{2}} d \eta_{2}^{(1)} \ldots \int_{q_{1}}^{q_{p}} d \eta_{p}^{(1)} \int_{\eta_{2}^{(1)}}^{\eta_{3}^{(1)}} d \eta_{3}^{(2)} \ldots \int_{\eta_{2}^{(1)}}^{\eta_{p}^{(1)}} d \eta_{p}^{(2)} \ldots \int_{\eta_{p-2}^{(p-3)}}^{\eta_{p}^{(p-3)}} d \eta_{\bar{p}}^{(p-2)} \int_{\eta_{p-2}^{(p-3)}}^{\eta_{p}^{(p-3)}} d \eta_{p}^{(p-2)} \int_{\eta_{p-1}^{(p-2)}}^{\eta_{p}^{(p-2)}} d \eta_{p}^{(p-1)} \\
& \times\left|\begin{array}{ccccccc}
L^{(0)(0)}\left(k_{1}, q_{1}\right) & L^{(0),(1)}\left(k_{1}, \eta_{2}^{(1)}\right) & \ldots & L^{(0),(\hat{p})}\left(k_{1}, \eta_{p}^{(\hat{p})}\right) & L\left(k_{1}, q_{p+1}\right) & \ldots & L\left(k_{1}, q_{n}\right) \\
L^{(1)(0)}\left(\xi_{2}^{(1)}, q_{1}\right) & L^{(1)(1)}\left(\xi_{2}^{(1)}, \eta_{2}^{(1)}\right) & \ldots & L^{(1)}(\hat{p})\left(\xi_{2}^{(1)}, \eta_{p}^{(\hat{p})}\right) & L^{(1)(0)}\left(\xi_{2}^{(1)}, q_{p+1}\right) & \ldots & L^{(1)(0)}\left(\xi_{2}^{(1)}, q_{n}\right) \\
\vdots & \vdots & & \vdots & \vdots & & \vdots \\
L^{\hat{p}, 0}\left(\xi_{p}^{(\hat{p})}, q_{1}\right) & L^{\hat{p}, 1}\left(\xi_{p}^{(\hat{p})}, \eta_{2}^{(1)}\right) & \ldots & L^{\hat{p}, \hat{p}}\left(\xi_{p}^{(\hat{p})}, \eta_{p}^{(\hat{p})}\right) & L^{(\hat{p}), 0}\left(\xi_{p}^{(\hat{p})}, q_{p+1}\right) & \ldots & L^{(\hat{p}),(0)}\left(\xi_{p}^{(\hat{p})}, q_{n}\right) \\
L^{(0)(0)}\left(k_{p+1}, q_{1}\right) & L^{(0)(1)}\left(k_{p+1}, \eta_{2}^{(1)}\right) & \ldots & L^{(p)(\hat{p})}\left(k_{p+1}, \eta_{p}^{(\hat{p})}\right) & L^{(0)(0)}\left(k_{p+1}, q_{p+1}\right) & \ldots & L^{(0)(0)}\left(k_{p+1}, q_{n}\right) \\
\vdots & \vdots & & \vdots & \vdots & & \vdots \\
L^{(0)(0)}\left(k_{n}, q_{1}\right) & L^{(0),(1)}\left(k_{n}, \eta_{2}^{(1)}\right) & \ldots & L^{(0),(\hat{p})}\left(k_{n}, \eta_{p}^{(\hat{p})}\right) & L^{(0)(0)}\left(k_{n}, q_{p+1}\right) & \ldots & L^{(0)(0)}\left(k_{n}, q_{n}\right)
\end{array}\right|
\end{aligned}
$$

Здесь $\widehat{p}=p-1$. Воспользовавшись оценкой (4.1) и неравенством Адамара, получим, что определитель в (4.2) допускает оценку

$$
\left(\prod_{m=1}^{p-1} m !\right)^{2} D^{n} n^{n / 2}
$$

Легко показать индукцией по числу $p$, что

$$
\int_{k_{1}}^{k_{2}} d \xi_{2}^{(1)} \ldots \int_{\xi_{2}^{(1)}}^{\xi_{3}^{(1)}} d \xi_{3}^{(2)} \ldots \int_{\xi_{p-1}^{(p-1)}}^{\xi_{p}^{(p-2)}} d \xi_{p}^{(p-1)}=\frac{1}{\prod_{m=1}^{p-1} m !} \prod_{p \geqslant i>j \geqslant 1}\left(k_{i}-k_{j}\right) .
$$

Отсюда и из оценки (4.3) следует, что при любом $\gamma \subset\{1, \ldots, n\}$

$$
\left|\operatorname{det}\left(L\left(k_{i}, k_{j}\right)\right)\right|<D^{n} n^{n / 2} \prod_{(i, j) \in \gamma}\left(k_{i}-k_{j}\right)^{2} .
$$

Заметим, что ядро $T_{n}^{\Lambda}\left(k_{1}, \ldots, k_{n}\right)$ представляется в виде

$$
T_{n}^{\Lambda}\left(k_{1}, \ldots, k_{n}\right)=\operatorname{det}\left(M_{\Lambda}\left(k_{i}, k_{j}\right)+1\right)-\operatorname{det}\left(M_{\Lambda}\left(k_{i}, k_{j}\right)\right)
$$


и к каждому из этих детерминантов в силу аналитичности функции $M_{\Lambda}\left(k_{1}, k_{2}\right)$ в области $W_{A} \times W_{A}$ применима доказанная оценка. Таким образом, лемма 3.2 доказана.

ЗАмЕчАниЕ. Лемма 3.2 (а также ее доказательство) обобщаются на случай любого числа попарно непересекающихся подмножеств $\gamma_{1}, \ldots, \gamma_{l}, \gamma_{i} \subset\{1, \ldots, n\}$, $i=1, \ldots, l$. Например, для случая двух подмножеств $\gamma_{1}, \gamma_{2}, \gamma_{1} \cap \gamma_{2}=\varnothing$,

$$
\left|\operatorname{det}\left(L\left(k_{i}, k_{j}\right)\right)\right| \leqslant D^{n} n^{n / 2} \prod_{(i, j) \subset \gamma_{1}}\left(k_{i}-k_{j}\right)^{2} \prod_{\left(i^{\prime}, j^{\prime}\right) \subset \gamma_{2}}\left(k_{i^{\prime}}-k_{j^{\prime}}\right)^{2} .
$$

\section{ЛиТЕРАТУРА}

1. Malyshev V. A., Minlos R. A. Invariant subspaces of clustering operators. I. J. Stat. Phys. 21, No. 3, 231-242 (1979); II, Commun. Math. Phys. 82, 211-226 (1981).

2. Малышев B. А., Минлос Р. А. Линейные операторы в бесконечно-частичных системах. Наука, М., 1992.

3. Onzager L., Kaufman B. Crystal statistics. Phys. Rev., 76, 232 (1949)

4. Минлос P. А., Синай Я. Г. Изучение спектра стохастических операторов, возникающих в решетчатых моделях газа. Теор. мат. физ., 2, 230-243 (1970).

5. Маматов ШІ. С., Минлос Р. А. Связанные состояния двухчастичного кластерного оператора. Теор. мат. физ., 79, №2, 163-179 (1989).

6. Маматов Ш. С. О связанных состояниях трансфер-матрицы гиббсовского решетчатого поля. Дисс. к.ф.-м.н., 1986.

7. Малышев B. А., Минлос Р. А. Гиббсовские случайные поля. Наука, М., 1985.

8. Abdullaev Zh. I., Lakaev S. N. On spectral properties of the matrix-valued Friedrichs model. Adv. Soviet Math., Vol. 5 (Minlos R. A., ed.), Amer. Math. Soc., Providence, R.I., 1991, pp. 1-37.

9. Minlos R. A., Zhizhina E. Leading branches of the transfer-matrix spectrum for lattice spin systems. J. Statist. Phys., 108, 885-904 (2002).

10. Abdullaev J., Minlos R. A. An extension of the Ising Model. Adv. Soviet Math., Vol. 20, Amer. Math. Soc., Providence, R.I., 1994, pp. 1-20.

11. Schor R. S., O'Carrol M. Transfer matrix spectrum and bound states for the lattice classical ferromagnetic spin systems at high temperature. J. Statist. Phys., 99, No. 5-6, 1265-1279 (2000).

12. Бирман М. Ш., Соломяк М. 3. Спектральная теория самосопряженных операторов в гильбертовом пространстве. Изд-во ЛГУ, Ленинград, 1980.

13. Яфаев Д. Р. Теория рассеяния. Изд-во СПбГУ, 1996.

14. Левин Б. Я. Распределение корней целых функций. Гостехиздат, М., 1956.

15. Лакштанов Е. Л. Старшие ветви спектра трансфер-матрицы для общих спиновых моделей с взаимодействием на один шаг. Работа принята к печати в Вестнике МГУ, сер. матем. (№6, 2004).

Московский государственный университет, email: alexenko@mccme.ru

Поступило в редакцию

Институт проблем передачи информации РАН, 8 апреля 2004 г. email: minl@iitp.ru 